\title{
The relative balance between languages predicts the magnitude of whole-language inhibition
}

\author{
Alba Casado 1, Jakub Szewczyk 1,2, Agata Wolna 1, \& Zofia Wodniecka 1 \\ 1. Psychology of Language and Bilingualism Lab, Institute of Psychology, Jagiellonian \\ University, Krakow, Poland \\ 2. University of Illinois at Urbana-Champaign, USA
}

Address for correspondence: Alba Casado, e-mail - alba.casado@uj.edu.pl; Zofia

Wodniecka, e-mail - zofia.wodniecka@uj.edu.pl; Psychology of Language and Bilingualism Lab, Institute of Psychology, Jagiellonian University in Krakow, Ul. Ingardena 6, 30-060

Authors' note: Upon acceptance, all raw data and the code needed to reproduce all results will be made publicly available.

Declaration of interest: none 


\title{
The relative balance between languages predicts the magnitude of
}

\author{
whole-language inhibition.
}

\begin{abstract}
After naming pictures in their second language (L2), bilinguals experience difficulty in naming pictures in their native language (L1). The "L2 after-effect" is a lingering consequence of inhibition applied to L1 to facilitate L2 production. We proposed that the amount of L1 inhibition depends on the relative balance between current activation of L1 and L2. In two experiments, bilinguals performed a blocked picture-naming task which provided a measure of the relative balance between the two languages and indexed whole-language inhibition via the magnitude of the L2 after-effect. The higher the activation level of L1 and the lower the activation level of L2, the bigger the L2 after-effect. The results also reveal an enduring down-regulation of L1 activation level in more language-balanced speakers. The outcomes support the main tenets of the inhibitory account of bilingual language production and indicate a high level of dynamics in the language system.
\end{abstract}

Keywords: whole-language inhibition; inhibitory control model; language balance; language activation level.

\section{INTRODUCTION}

In speakers who know more than one language, all the languages remain active in their mind even when one language alone is required in a given context. The phenomenon of language co-activation has been observed in written word recognition (e.g., Dijkstra, 2005), in spoken word recognition (Marian \& Spivey, 2003), in written production (Iniesta, Paolieri, Serrano, \& Bajo, 2020), and in spoken production (e.g., Kroll, Bobb, \& Wodniecka, 2006). The remarkable discovery of language co-activation has guided subsequent research in the field that attempts to characterize how bilinguals are able to successfully use contextually appropriate language, despite the ubiquitous language non-selectivity and consequent interference. Previous research focused on understanding which mechanisms regulate the co- 
activation and interference from the unwanted language(s). The most popular explanatory framework points to language inhibition as the key regulatory process (Green, 1998), although there is a growing notion that more than one control mechanism is probably involved in bilingual language processing (e.g., Gollan, Schotter, Gomez, Murillo, \& Rayner, 2014). Still, the precise nature of the language control mechanisms is poorly understood. It is also an open question whether all bilinguals use the same mechanisms at all times, or depending on the particular combination of language experiences of each bilingual (e.g., language pairing, context of language use, relative balance in proficiency between the languages, etc.) - whether specific mechanisms of control are involved (e.g., Declerck, Kleinman, \& Gollan, 2020; Van Assche, Duyck, \& Gollan, 2013).

In the current paper, we focus on one potential control mechanism: whole-language inhibition (Van Assche et al., 2013)1. In particular, we test if individual variations in bilinguals' relative balance between two languages impacts the magnitude of whole-language inhibition during language production.

\section{Inhibitory account of bilingual language use}

As already indicated above, the inhibitory account is the most viable framework that explains how bilinguals can successfully use the language that is appropriate in a given context and resist interference from the unwanted language. It was formulated by Green (1998) in the Inhibitory Control Model (IC). The IC account assumes that in order for the weaker language (L2) to be used, the stronger language (L1) needs to be inhibited. Inhibition applied to L1 lasts for some time after L2 use is finished and consequently leads to a slowdown of L1 retrieval following L2 production.

\footnotetext{
1 Sometimes referred to as "global inhibition" (e.g., Abutalebi \& Green, 2016)
} 
Most empirical evidence that is interpreted as supporting the IC model comes from studies that employed a language-switching paradigm. In this paradigm, participants are asked to switch from naming in one language to naming in another on a trial-to-trial basis (for a review, see Bobb \& Wodniecka, 2013; Declerck \& Philipp, 2015; Kleinman \& Gollan, 2018). The typical (although not univocal) outcome is that there is a larger cost of switching to the stronger language than into the weaker language: the return to the stronger language after using the weaker language is more costly than the return to the weaker language after using the stronger language. This so-called language-switching cost asymmetry has been suggested to reflect the cost of reactivating L1, which was inhibited in the previous naming trial in L2 (Meuter \& Allport, 1999)².

Another source of evidence supporting the IC model comes from studies that used a blocked picture-naming paradigm rather than a language-switching paradigm. This paradigm allows "the L2 after-effect" to be measured; this phenomenon consists of lingering increased difficulty in lexical access in the native language following the use of the second, weaker language. The blocked picture -naming paradigm allows the L2 after-effect to be measured by comparing the processing costs of the production of the native or stronger language when it follows longer (blocked) production in a second or a weaker language, compared to when it follows production in the native language (e.g., Branzi, Martin, Abutalebi, \& Costa, 2014; Wodniecka, Szewczyk, Kałamała, Mandera, \& Durlik, 2020). In experiments focused on the L2 after-effect in which the blocked picture-naming paradigm was used, participants had to

\footnotetext{
2 An alternative account explains the language switching cost asymmetry by assuming persisting activation (enhancement) of the weaker language rather than inhibition of the stronger language (Philipp, Gade, \& Koch, 2007). Under this account, when bilinguals have to speak in their nondominant language (L2), the L2 has to be strongly activated in order to surpass the stronger residual activation of the dominant language (L1). Consequently, when the bilinguals are required to switch from speaking in L2 to speaking in L1, the strong residual activation of the previously activated L2 will hinder speaking in L1. Disentangling between the L2 activation/enhancement or the L1 inhibition accounts is, however, outside of the scope of the current study.
} 
name pictures either in L1 or L2, with the language of naming changing between blocks (Misra, Guo, Bobb, \& Kroll, 2011; Branzi et al., 2014; Wodniecka et al., 2020). For instance, in a recent study by Wodniecka and colleagues (2020), Polish (L1) learners of English (L2) named pictures in L1 following the naming of pictures in either L1 or L2. The results showed a slow-down of response latencies when pictures were named in L1 just after they were named in L2. Interestingly, the effect persisted for at least 5 minutes after changing the language. Similar lingering consequences of L2 use on subsequent L1 use have been recently observed for naming pictures in L1 after reading aloud in L2 (Degani, Kreiner, Ataria, \& Katheeb, 2020); however, they have also been observed in other experimental paradigms, such as the n-2 paradigm (Declerck, Thoma, Koch, \& Philipp, 2015; Declerck \& Philipp, 2018; Guo, Liu, Chen, \& Li, 2013).

The IC model assumes that inhibition is reactive, which means that the amount of inhibition needed to suppress non-target lexical representations (i.e., lemmas with the incorrect language tag) is proportional to their initial activation level ${ }^{3}$. Although it is not explicitly stated in the IC model, this implies that the amount of inhibition applied to the lemmas in the non-target language should vary from speaker to speaker, depending on the relative balance in the activation levels between L1 and L2 lemmas. The consequence of this assumption is that the strength of inhibition should depend on the relative balance between the languages known by a given speaker. Following Degani and colleagues (2020), we assume that the relative language balance depends on the default activation level of a given language (related to general fluency in this language and current immersion experience) and on more immediate language-activation demands (related, e.g., to a recent language use). In speakers

\footnotetext{
${ }^{3}$ More specifically, as stated by Green (1998) "because inhibition is reactive, more active lemmas will be more inhibited" (p. 74)
} 
who are clearly dominant in one language, the dominant language (L1) should have a higher default level of activation than the weaker language (L2) and thus a larger amount of inhibition needs to be applied to L1 lemmas when L2 is used. In speakers who have comparable activation levels in both languages (i.e., balanced bilinguals), the inhibition applied to any of the languages of a bilingual should be very small and mostly absent (see Figure 1).

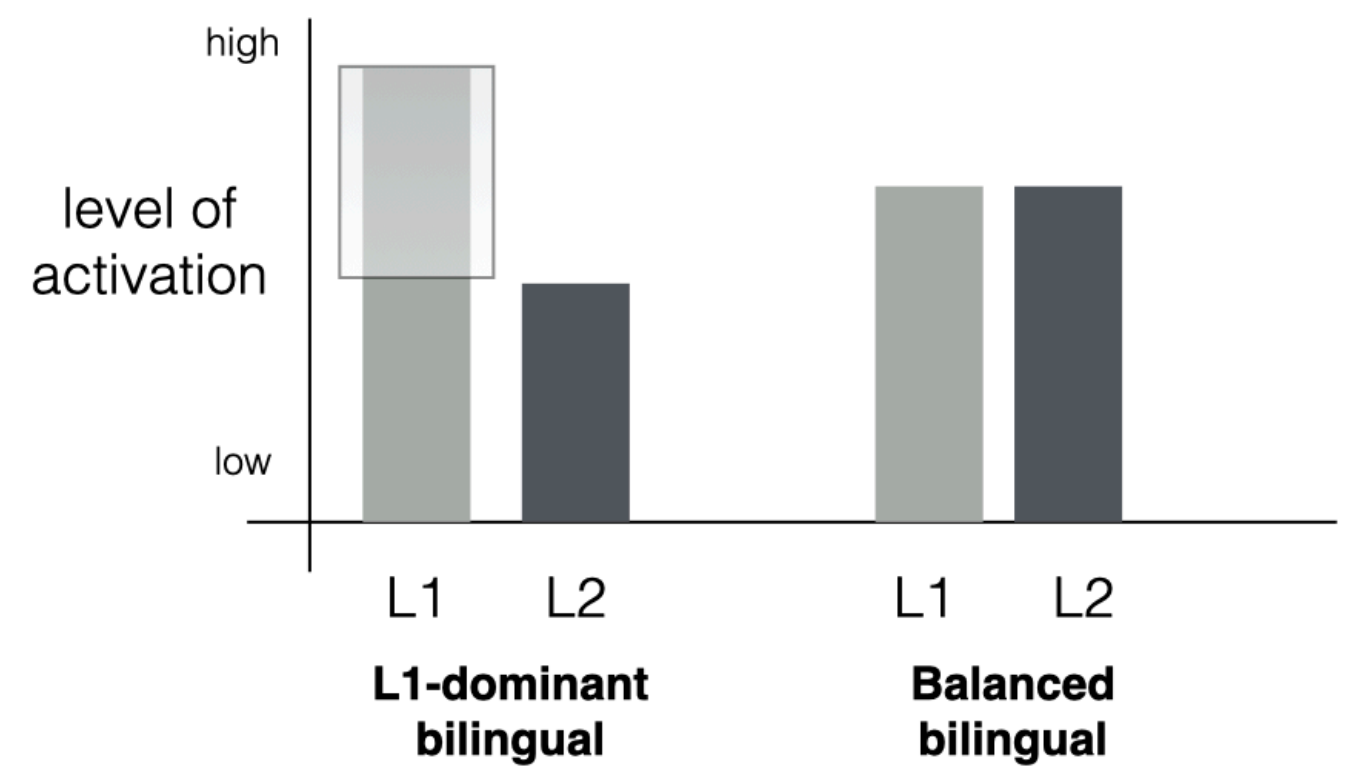

amount of inhibition applied to L1

Figure 1. Symbolic representation of two ideal speakers with a strong difference in language balance. The graph illustrates the amount of inhibition that an L1-dominant bilingual needs to apply to L1 to access L2 (left), compared to a perfectly balanced bilingual (right).

Inhibition as a function of the degree of current language activation

Here, we propose a causal model that outlines the assumptions of the IC account with respect to the current activation level of a bilingual's languages. We present the model using a 
Directed Acyclic Graph (DAG; Pearl, 2018) which makes explicit assumptions about the causal relations between theoretical constructs and measures. The model (hereafter referred to as the IC-b model) describes the situation in which language-activation levels change due to requirements in the environment (specifically, the required use of L2). In the presented model, we consider the relative balance of two languages in two different time points in which the participants were tested (see Figure 2).

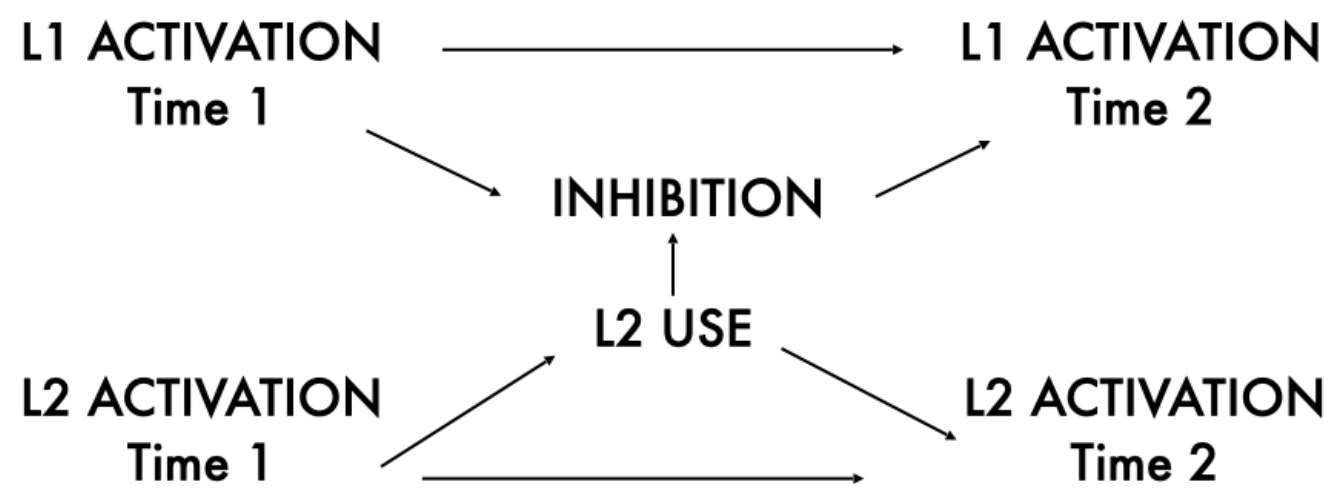

Figure 2. DAG model representing how the relative language balance (activation level of L1 and L2 at Time 1) and involvement of inhibition during L2 use changes the relative language balance (activation level of L1 and L2 at Time 2). The activation of L1 and L2 at Time 2 depends on both the current activation levels (i.e. it is directly related to the relative balance of a bilingual speaker) and the immediate language activation demands due to contextual requirements (e.g., using L2), which trigger the inhibitory mechanisms.

The proposed IC-b model explains how L1 activation changes between two time points (Time 1 vs. Time 2) due to manipulation involving L2 use. At Time 1, namely when a 
bilingual speaker is immersed in her natural language environment, she has a certain default level of L1 and L2 activation (see Figure 2). If, at some point, she is asked to use her L2, this leads to an increase of the activation level of all lemmas in L2. Critically, the use of L2 triggers an inhibitory mechanism that dampens the activation of lemmas in L1. The amount of inhibition depends on the current activation (measured at Time 1) of lemmas in both L1 and in L2. When the current activation level of lemmas is balanced, the same amount of inhibition should be applied to L1 (when L2 is used) as to L2 (when L1 is used). The more the L1 lemmas are activated compared to L2 lemmas, the higher the amount of inhibition that is applied to L1. Thus, when the activation of L1 and L2 lemmas is measured in languageimbalanced speakers again after L2 use (Time 2), the activation level of L1 lemmas should be lower, while the activation level of L2 lemmas should be higher, compared to a situation in which no L2 was used between Time 1 and Time 2. In short, the more balanced a speaker is, the smaller the difference between the activation of L1 and L2 lemmas at Time 1, and also at Time $2^{4}$.

Moreover, the IC-b model assumes that this inhibition will persist for some time after the L2 use, thus keeping the activation of L1 lemmas low even if a bilingual speaker returns to using her L1. This assumption is consistent with data indicating that difficulty in accessing L1 lemmas after using L2 is relatively long-lasting: it has been shown to persist over at least a couple of minutes after a language switch (Branzi et al., 2014; Wodniecka et al., 2020).

Within the current study, we present two experiments which test the specific predictions of the IC-b model. Before discussing the experiments in detail, we briefly present

\footnotetext{
4 Some researchers claim that balanced bilinguals do not need to apply any inhibition at all (Costa \& Santesteban, 2004; Schwieter \& Sunderman, 2008). Our causal model is also compatible with such scenarios.
} 
the available evidence on the relation between the magnitude of inhibition applied and the individual relative balance between the two languages.

\section{Empirical evidence on the relation between language activation and inhibition}

Several studies support the idea that the degree of language balance determines the amount of inhibition. Within the most commonly used paradigm (language switching) two indices of inhibition have been used to establish the relation between the degree of language balance and the magnitude of inhibition: language-switching cost asymmetry (described above; for a review see Bobb \& Wodniecka, 2013), and more recently, the reversed dominance effect, i.e., an overall, temporary slowdown of naming in L1 in a language-mixing context (Declerck et al., 2020). However, as argued in Bobb and Wodniecka (2013), the language-switching paradigm may not be ideal for deriving an index of inhibition. Because bilinguals do not switch between languages from one word to another on a daily basis, the requirements emulated in the language-switching task are highly unnatural. In other words, the language-switching paradigm may not tap into processes that are typically engaged in bilingual language processing. Moreover, the language-switching paradigm typically involves multiple repetition of items (e.g., in Costa \& Santesteban's (2004) study, in Experiment 1, 10 items were repeated across 950 trials), and it has been shown that such repetition enhances lexical access in L1 and L2 differently (Kleinman \& Gollan, 2018). In consequence, the repetition of items likely impacts the difficulty of returning to L1 after using L2 in a way that is difficult to control.

Regarding the index of language balance, most past studies have relied on L2 proficiency as a proxy for the language balance. An implicit assumption is that high proficiency in L2 implies a greater balance between the languages. Some authors measured 
L2 proficiency with a competence test (Filippi, Karaminis, \& Tomas, 2014), while others used self-assessment questionnaires (Costa \& Santesteban, 2004; Costa, Santesteban, \& Ivanova, 2006; Lu et al., 2019), and others used verbal fluency tasks in L2 (Schweiter \& Sunderman, 2008). Altogether, the studies found that the lower the L2 proficiency (or the lower the L2 verbal fluency), the larger the cost of switching to L1. This finding is in line with the idea that the degree of language balance is related to the magnitude of inhibition applied.

An important limitation of this line of research is that language balance is assessed indirectly by relying on proficiency measures, and specifically only on L2 proficiency (e.g., self-ratings, test of grammar abilities or vocabulary). However, the initial IC proposal (Green, 1998) assumes that the amount of inhibition depends on the relative balance in the activation of both languages. Importantly, language activation is not necessarily related to L2 proficiency, but it probably depends on other contextual factors like immersion or a recent language use.

Some exceptions from the line of research described above (where language balance was assessed indirectly based only on L2 proficiency) are two studies which proposed different ways of measuring the language balance. The first one is a recent study by Declerck and colleagues (2020), in which the authors used an index of language balance that relied jointly on both L1 and L2 proficiency instead of relying on L2 proficiency only. More specifically, the authors used the Multilingual Naming Test (Gollan, Weissberger, Runnqvist, Montoya, \& Cera, 2012), which allows the language-balance index to be derived from picture-naming accuracy in L1 and L2. While we believe that combining both L1 and L2 proficiency preserves the idea that language balance must be measured by focusing on both rather than one language, accuracy in a picture-naming task does not directly reflect the level of language activation. Response latencies, however, can reflect more direct and precise 
differences in language activations: by providing fine measurement on a scale of milliseconds they offer much better "resolution" to measure the ease of accessing and producing a given word.

The second study that measured language balance without using L2 proficiency is a pioneering study by Meuter and Allport (1990). In this study, the authors used response times to measure the balance between activation levels in the two languages. In particular, the authors assessed the default language activation by calculating the difference between participant's speed of naming numerals in L1 and L2. They found that when bilinguals were unbalanced (big differences between L1 and L2 naming speed), the magnitude of the switching cost was bigger for switching to L1 compared to switching to L2; when bilinguals were more language-balanced (small differences between L1 and L2 naming speed), the magnitude of the switching cost was similar for switching to L1 and to L2. In other words, with a comparable activation of the two languages, the switching cost asymmetry disappeared. Meuter and Allport interpreted their results as indicating that to successfully use their L2, unbalanced bilinguals need to apply more inhibition to L1 than balanced bilinguals.

All in all, most evidence for the relationship between language inhibition and individual language balance is limited due to two methodological issues: (1) using an index of language inhibition that relies on the language-switching paradigm, and (2) using an indirect index of language balance, usually L2 proficiency. Moreover, most of the previous evidence is based on a confounded assessment of how broadly the inhibition might affect the language system, i.e., whether it affects the whole language, a specific category of items, or specific items. In the two experiments presented below, we addressed these methodological shortcomings by using a blocked picture-naming paradigm and focusing on pictures that participants only name once in the course of the experiment. As such, this paradigm allows 
purer measurement of the (whole-) language inhibition than the language-switching paradigm. It also affords a more direct test of the degree of an individual's balance between the two languages.

\section{The current study}

The goal of the two experiments reported in this paper was to test the predictions of the IC-b model presented above. In each of the experiments, we tested a different group of unbalanced Polish-English speakers. In both experiments we used a blocked picture-naming paradigm which allowed us to address the methodological issues discussed in the previous sections. This paradigm makes it possible to trace within-speaker changes in languageactivation levels across different time points. In this paradigm, participants are asked to name pictures in language-consistent blocks (L1 or L2). They first complete a baseline naming block (in L1 or in L2) followed by an experimental block of naming in L1. The critical comparison involves experimental blocks of L1 naming after baseline naming in L1 vs. after baseline naming in L2. In our previous study that employed this paradigm (Wodniecka et al., 2020), we reported an "L2 after-effect", i.e., slower L1 naming latencies after using L2 compared to after using L1. Moreover, the L2 after-effect was observed for completely new items, and therefore it appears to reflect control mechanisms applied over the entire language. Therefore, we propose that the "L2 after-effect" can be used as a proxy of the magnitude of L1 inhibition on the whole-language level.

Following the predictions of the IC-b model, we assumed that a larger difference between L1 naming after L2 (compared to L1 naming after L1) is indicative that a greater amount of L1 inhibition is applied during L2 use. To index the relative balance in activation between the two languages for a given individual, we used a continuous measure which was 
based on the difference between participants' mean baseline naming latencies in L1 and L2 naming. Importantly, both inhibition and balance indices were independent because they were derived from different blocks of the task (see details in the Method section below). Overall, the design of the study allowed us to simultaneously measure the current activation levels of L1 and L2 as well as the amount of (whole-language) inhibition applied to L1 as a result of using L2. Experiment 2 aimed to replicate the findings of Experiment 1 and additionally control for potential confounds identified in Experiment 1.

\section{EXPERIMENT 1}

For Experiment 1, we reanalyzed the data from our earlier study (Wodniecka et al., 2020), in which a relatively large group of bilinguals performed a battery of language and cognitive tasks. The testing was done three times across the span of two years. In the present reanalysis we focus on the behavioral data from the picture naming task obtained in the first session 5 . Here, we reanalyzed the behavioral data from the picture-naming task in which participants named pictures in two testing stages, separated by 30 minutes of other tasks completed in L1. Within each testing stage, there were two naming blocks of 60 pictures each. In both testing stages, the second block involved naming in L1, while the first blocks differed with respect to the language used in naming pictures: in testing stage 1 it was L1, and in testing stage 2 it was L2 (see Figure 3 for an overview of the design).

As the index of inhibition, we used the L2 after-effect, defined as the difference in L1 naming latencies between the second block of the first testing stage (i.e., L1 naming after L1)

\footnotetext{
5 The reanalysis of behavioral data from picture naming obtained in the other two sessions is presented in Appendix A. The pattern of responses both in session 2 and session 3 replicates the findings of session 1 presented here. In the original study we also collected EEG data but it will be presented elsewhere.
} 
and the second block of the second testing stage (i.e., L1 naming after L2). Our previous analysis of this dataset (Wodniecka et al., 2020) showed that L1 naming latencies measured in the second block were longer when they were preceded by L2 naming, compared to when preceded by L1 naming. In the present analysis, we ask a new question concerning whether the size of the L2 after-effect depends on language balance.

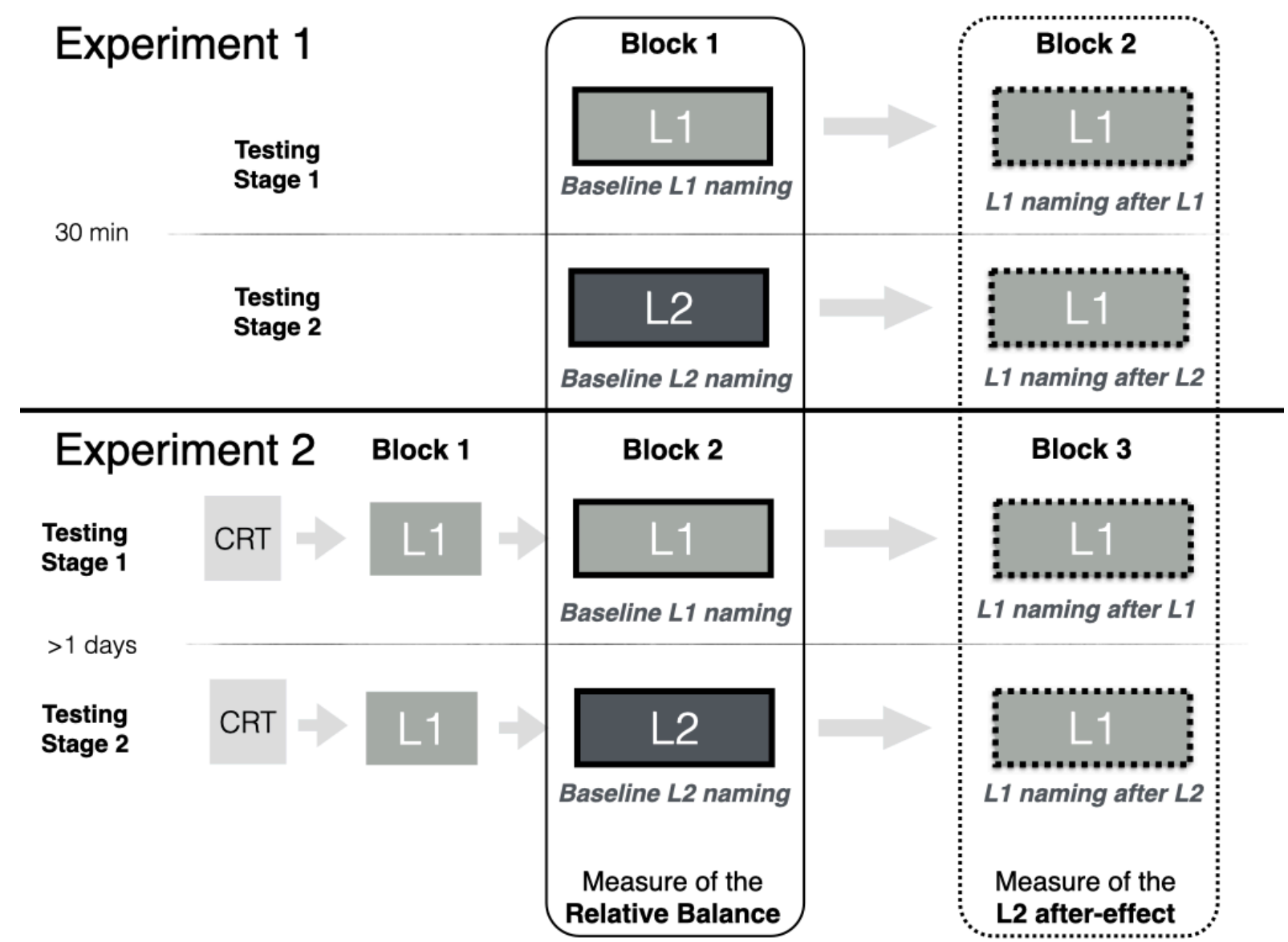

Figure 3. Schematic representation of the task's design. In Experiment 1, two testing stages were separated by 30 min.; at each stage, participants completed 2 blocks of a picture-naming task. In Experiment 2, the two testing stages were separated by at least 1 day; in each stage, participants first performed a Choice Reaction Time task (CRT), followed by three different blocks of Picture Naming

To estimate the balance between L1 and L2 for each participant, we focused on the first blocks in which participants named pictures in L1 and in L2. We assumed that the mean naming latencies in the first blocks can serve as a proxy for estimating the activation level of 
L1 and L2 lemmas, and subsequently, the relative language balance of each participant. Importantly, so the estimates of language balance would not be confounded with the L2 aftereffect, the data from the first blocks were not used in the estimation of the L2 after-effect.

The design of the study (Figure 3) maps quite straightforwardly to the causal model of L2 after-effects outlined earlier (Figure 2). L1 and L2 activation level at Time 1 is measured in the first blocks of each testing stage (from now on, we will refer to them as the baseline naming latencies). At the same time, naming pictures in L2 in the first block of the second testing stage serves as the "L2 use" experimental manipulation. We can appreciate its effect on L1 activation at Time 2 (which, according to the theory should be reduced by the engagement of inhibition) by comparing L1 naming latencies in the second blocks of each testing stage. Note that in our design the L2 activation at Time 2 is not measured.

\section{Predictions based on the proposed IC-b model}

We expected that the smaller the difference between the baseline naming latencies in L1 and L2 (indicating that a participant is more language balanced), the smaller the L2 aftereffect would be. Analogously, the lower the baseline naming latencies in L1 relative to L2 naming latencies (indicating that L1 baseline activation is higher than L2), the bigger the L2 after-effect should be. In order to operationalize the hypotheses based on the IC-b model (see Fig. 2), which assumes that the L1 and L2 baseline activation levels can independently regulate inhibition, we predicted that the magnitude of the L2 after-effect would be negatively associated with baseline naming latencies in L1, and positively associated with baseline naming latencies in L2. 


\section{METHODS}

\section{Participants}

Eighty-four students from three high schools in Kraków, Poland volunteered to participate in the study. Eight participants were removed due to various technical problems or incomplete data, leaving 76 participants who entered the analyses (mean age $=16.4$ years, SD $=0.5 ; 54$ female). The experiment met the requirements of the Ethics Committee of the Institute of Psychology of Jagiellonian University concerning experimental studies with human subjects. Since our participants were minors, written informed consent for participation was given both by the participants and by their parents prior to the experiment. After completing the experimental session, all participants received small gifts and were included in a prize lottery. Background information on participants' self-assessed language proficiency, learning history and extent of language use were collected with a language background questionnaire based on a language history questionnaire (Li, Sepanski, \& Zhao, 2006). For all the participants, Polish was the only language acquired in early childhood. They started learning English by the age 6, and began using it more intensively when they started attending secondary school at around the age of 13. All participants declared they had started learning another foreign language (L3; predominantly German or French), but their L3 proficiency was low and they did not speak L3 outside the classroom context. All participants reported using mostly L1 during an average day and speaking L2 only occasionally, mostly during English lessons at school. Similarly, participants considered their L1 proficiency significantly higher than L2 (for detailed self-assessment data see Table 1). To obtain an objective L2 proficiency measure, we also asked participants to fill in the Lexical Test for Advanced Learners of English, LexTALE (Lemhöfer \& Broersma, 2012). On average, participants obtained a score of $67 \%(\mathrm{SD}=11 \%)$, indicating that they were moderately 
proficient in their L2. Table 1 shows L1 and L2 picture-naming accuracy and naming latencies at baseline, thus indicating a clear relative dominance of their L1. To ensure that faster naming latencies in L2 were associated with higher accuracy scores in L2 naming, we run a correlation analysis between the two measures at baseline (Block 1); the results showed a negative correlation, $\mathrm{r}=-.024 ; \mathrm{p}=.037$, thus showing that participants who were faster in L2 were also more accurate in L2. We also assessed their semantic verbal fluency scores both for L1 and L2 (participants produced as many words as possible in $60 \mathrm{~s}$ in each language; the categories were animals, fruits \& vegetables, and body parts, rotated across the participants and languages). Participants produced significantly more words belonging to the target semantic category in L1 (mean $=21.8, \mathrm{sd}=5)$ than in L2 $($ mean $=15.3, \mathrm{sd}=5.3)$.

EXPERIMENT 1

EXPERIMENT 2

$\begin{array}{llll}\text { L1 } & \text { L2 } & \text { L1 } & \text { L2 }\end{array}$

\begin{tabular}{lcccc}
\hline $\begin{array}{l}\text { Self-rated proficiency (1- } \\
7)\end{array}$ & $6.93(0.16)$ & $5.2(0.7)$ & $9.86(0.1)$ & $7.65(0.2)$ \\
\hline Percentage of daily use (\%) & $79.7(15.7)$ & $16(12.8)$ & $80(26)$ & $16(13)$ \\
\cline { 4 - 5 } & - & $66.97(10.64)$ & $72.8(7.1)$ & $72.7(20.4)$ \\
\hline $\begin{array}{l}\text { Lextale (mean accuracy in } \\
\%\end{array}$ & $93.5(24.6)$ & $75.2(43.9)$ & $97.1(16.9)$ & $89.2(31)$ \\
\hline $\begin{array}{l}\text { Baseline naming accuracy } \\
(\%)\end{array}$ & $889(249)$ & $1149(317)$ & $970(284)$ & $1165(338)$ \\
\hline $\begin{array}{l}\text { Baseline naming latencies } \\
(\mathrm{ms})\end{array}$ & & & & \\
\hline
\end{tabular}

Table 1. Participants' characteristics of the Polish-English bilinguals in Experiment 1 and 2. Note: Standard deviations are given in parenthesis. Self-rated measures based on self-assessment questionnaire: self-rated proficiency (rated on a scale 1-7 in Experiment 1, and from 1-10 in Experiment 2); percentage of daily use. Originally they were asked to rate the percentage of daily use of L1, L2 and L3. In the present table we only include the ratings for L1 and L2 (in Experiment 2 separately for active use (top) and passive use (bottom) - this distinction was not available for Experiment 1). Objective measures: Lextale. Experimentally-derived measures from the picture naming task: Baseline naming accuracy and Baseline naming latencies. 


\section{Materials}

Stimuli in the picture-naming task included 180 pictures selected from the online database of the International Picture Naming Project (Szekely et al., 2004). All pictures were black-and-white line drawings sampled from a range of semantic categories; no PolishEnglish cognates were included. The pictures were divided into six bins of 30 pictures, each matched in visual complexity, length of the most common names in Polish and English, age of acquisition in English, and the frequency of use in Polish and English (Polish: SUBTLEXPL; Mandera, Keuleers, Wodniecka, \& Brysbaert, 2015; English: SUBTLEX-US; Brysbaert \& New, 2009). The six sets were rotated across the experimental conditions such that each picture occurred in each condition across participants.

\section{Procedure}

Each trial began with the presentation of a blank screen with randomized duration (700-1300 ms) followed by the presentation of a fixation point in the center of the screen for $1000 \mathrm{~ms}$. Subsequently, the fixation point was replaced by the target picture until the timeout (3000 ms) or a response occurred. The stimuli were presented using DMDX software (Forster \& Forster, 2003) on a 17-in. screen positioned approximately $80 \mathrm{~cm}$ from the participant. Participants were instructed to name pictures aloud as quickly and accurately as possible. Reaction times for vocal responses were recorded; naming latencies were automatically measured using a DMDX voice key and subsequently manually screened for any non-speech sounds. The task was administered in two testing stages, separated by other tasks in L1 for approx. 30 minutes (see Figure 1 above). Within each testing stage, there were two blocks of picture naming with 60 pictures each. The blocks of L1 naming were preceded by a block of naming in either L1 (first block of the first testing stage) or L2 (the first block of the second testing stage; see Figure 3). Half of the pictures used in the second blocks of each testing 
stage were new, and half were repeated from the preceding block. The information concerning the target language for each block was presented at the onset of the block. Participants completed 8 practice trials before the onset of the first block in each testing stage. Participants were asked to take a short break between the first and the second block of each testing stage. Each of the blocks lasted approximately 10 minutes. Overall, participants named 180 unique pictures, 60 of them twice (the repeated conditions). For the purposes of the present study, we analyzed the new items exclusively ${ }^{6}$.

\begin{tabular}{|c|c|c|c|c|c|}
\hline & \multirow{2}{*}{\multicolumn{2}{|c|}{ Baseline block }} & & \\
\hline & & & & \multicolumn{2}{|c|}{ Experimental block } \\
\hline \multirow{4}{*}{ EXPERIMENT 1} & \multirow[b]{2}{*}{ Testing Stage } & \multicolumn{2}{|c|}{ Block 1} & \multicolumn{2}{|c|}{ Block 2} \\
\hline & & Language & Mean (SD) & Language & Mean (SD) \\
\hline & Stage 1 (L1-L1) & $\mathrm{L} 1$ & $.94(.24)$ & L1 & $.95(.08)$ \\
\hline & Stage 2 (L2-L1) & L2 & $.75(.44)$ & L1 & $.94(.06)$ \\
\hline \multirow{4}{*}{ EXPERIMENT 2} & & \multicolumn{2}{|c|}{ Block 2} & \multicolumn{2}{|c|}{ Block 3} \\
\hline & Testing Stage & Language & Mean (SD) & Language & Mean (SD) \\
\hline & Stage 1 (L1-L1) & L1 & $.97(.17)$ & L1 & $.97(.17)$ \\
\hline & Stage 2 (L2-L1) & L2 & $.89(.31)$ & L1 & $.96(.18)$ \\
\hline
\end{tabular}

Table 2. Mean accuracy in picture naming in the baseline and experimental blocks calculated by-participant.

\section{Data analysis}

Prior to running the analyses on naming latencies, for each participant we removed all trials with errors, timeouts, as well as trials with recordings that were too quiet to recognize the response. All response latencies were filtered to include only the 300-2000 ms range.

\footnotetext{
${ }^{6}$ In the present model we only take into account the whole-language level of inhibition assessed by new-items. The item-level source of inhibition (which could be assessed by analyzing repeated items) is out the scope of the present study.
} 
They were also transformed using a reciprocal transformation $(-1000 / \mathrm{RT})$ due to the rightskewed distribution of the data.

\section{Statistical analyses}

All analyses were performed using linear mixed-effects models, as implemented in the lme4 package (version 1.1.21; Bates, Mächler, Bolker, \& Walker, 2015) in R (R Core Team, 2017), using participants and pictures as crossed random effects.

\section{Indices of L1 and L2 activation at Time 1}

The indices of L1 and L2 activation at Time 1 (see Figure 2) were estimated based on the mean naming latencies in L1 and L2 in the first blocks of both testing stages (see Figure 3). To maximize the reliability of these indices, instead of just computing the mean naming latency for each participant, we ran two mixed-effects regression models, one for L1 and another for L2, in which the dependent variable was naming latency in the first blocks. In this model we had no predictors except the intercept. We also included by-subject and by-item random intercepts. Next, we extracted the models' per-participant estimates of naming latency in L1 and L2 (the models' intercept, modified by the appropriate by-Participant random intercept; see www.osf.io/27y4p for details). This way of estimating the by-Participant naming latencies ensured higher reliability because the mixed-effects model accounted for the regression to the mean. We will refer to these estimates as the Estimate RT of L1 naming and the Estimate RT of L2 naming.

\section{The main model}

In the main model, the dependent variable was L1 naming latency in the second blocks of both testing stages (see Figure 3). As fixed effects we included Preceding Language, Estimate RT of L1 naming, Estimate RT of L2 naming, interaction between the Preceding Language and Estimate RT of L1 naming, and interaction between the Preceding Language and Estimate 
RT of L2 naming. We also included (log-transformed) Trial number as a predictor to control for effects of familiarization, fatigue, or cumulative semantic interference that could accumulate during the experiment. The categorical predictor of Preceding Language was deviation coded (preceding L1 $=-0.5$, preceding $\mathrm{L} 2=0.5$ ) and the continuous predictors (Trial number, Estimate RT of L1 naming, and Estimate RT of L2 naming) were centered prior to running the analyses. We fitted the maximal model first (Barr, Levy, Scheepers, \& Tily, 2013), and in the case of non-convergence or singularities we simplified it following the recommendations outlined in Bates and colleagues (2015). The final model included the bysubject random intercept and uncorrelated random slope for Preceding Language. It also included by-item random intercept and uncorrelated random slope for the interaction between Estimate RT of L1 naming and Preceding Language. We considered as significant any fixed effect with an absolute t-statistic value higher than 2 .

\section{RESULTS}

Table 2 shows the accuracy in all blocks of the task. Overall, participants were more accurate when naming in L1 than naming in L2; descriptively, the accuracy of L1 naming was the same in all blocks and testing stages.

The analysis of naming latencies in the second blocks of both testing stages showed a significant fixed effect of Preceding Language (i.e., the L2 after-effect). Participants were slower to name pictures in L1 when the naming followed a block of naming in L2, compared to when it followed another block of naming in L1. In addition, there was a significant effect of Estimate RT of L1 naming (the faster participants named pictures in L1 in the first block, the faster they named pictures in L1 in the second blocks of both testing stages), and of Estimate RT of L2 naming (the faster participants named pictures in L2 in the first block, the 
slower they named pictures in L1 in the second blocks of both testing stages).

\begin{tabular}{llllll}
\hline EXPERIMENT 1 & & & & & \\
Effect & Estimate & $\mathrm{SE}$ & $\mathrm{t}$ & by-Item SD & by-Subject SD \\
\hline Intercept & -1.21 & 0.02 & -63.52 & 0.12 & 0.05 \\
Preceding.Lang & 0.10 & 0.01 & 8.73 & & 0.07 \\
Estimate.RT.L1.naming & 0.67 & 0.06 & 10.80 & & \\
Estimate.RT.L2.naming & 0.32 & 0.08 & 3.81 & & \\
Trial.number & 0.09 & 0.02 & 5.18 & & 0.02 \\
PrecedingLang:Estimate.RT.L1.naming & -0.33 & 0.09 & -3.43 & 0.14 & - \\
PrecedingLang:Estimate.RT.L2.naming & 0.39 & 0.13 & 3.04 & & - \\
\hline EXPERIMENT 2 & & & & & \\
Effect & Estimate & $\mathrm{SE}$ & $\mathrm{t}$ & by-Item SD & by-Subject SD \\
\hline Intercept & -1.10 & 0.01 & -90.00 & 0.14 & 0.06 \\
PrecedingLang & 0.03 & 0.01 & 5.03 & & \\
Estimate.RT.L1.naming & 0.58 & 0.08 & 6.56 & 0.27 & - \\
Estimate.RT.L2.naming & 0.29 & 0.11 & 2.50 & & - \\
Estimate CRT & 0.09 & 0.02 & 4.02 & - & \\
LogTrialNumber & -0.09 & 0.00 & -1.78 & & \\
Session & 0.01 & 0.01 & 1.73 & & \\
PrecedingLang:Estimate.RT.L1.naming & -0.18 & 0.06 & -2.91 & 0.20 & - \\
PrecedingLang:Estimate.RT.L2.naming & 0.25 & 0.07 & 3.43 & & - \\
\hline & & & & & \\
\hline
\end{tabular}

Table 3. Fixed and random effects from the LME model of naming latencies in the experimental blocks (Block 2 of Experiment 1 and Block 3 of Experiment 2).

Crucially, Preceding Language entered into two significant interactions: with Estimate RT of L1 naming and with Estimate RT of L2 naming (see Table 3). The L2 after-effect was dependent on naming latencies of both L1 and L2 in the baseline blocks: it was larger for participants who were faster in L1 naming at baseline (in the first block) and slower in L2 naming at baseline (in the first block). In other words, the L2 after-effect was larger for participants whose naming latencies in block 1 showed the largest imbalance between L1 and L2 in favor of faster naming in L1 (see Figure 4). Finally, there was an effect of Trial number, indicating that as the experiment progressed, participants were slower to name the pictures. 


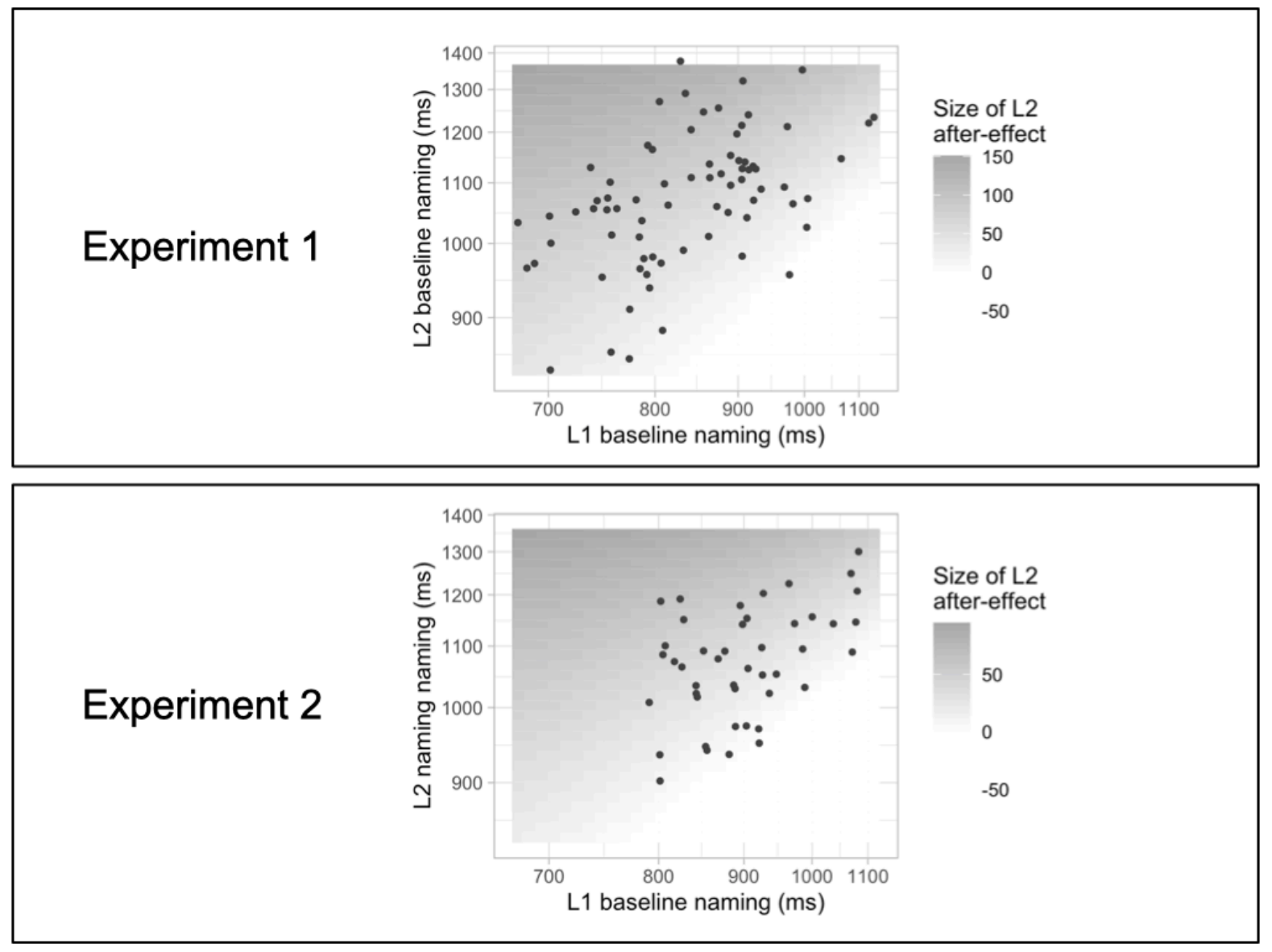

Figure 4. Model-based predictions of the magnitude of the L2 after-effect as a function of L1 and L2 baseline naming speed in Experiment 1 and Experiment 2. The X-axis corresponds to L1 baseline naming latencies (transformed back to milliseconds) measured in block 1, while the y-axis corresponds to L2 baseline naming latencies (transformed back to milliseconds). The black points show L1 and L2 baseline naming latencies of the participants. The L2 after-effect was calculated by subtracting the predicted naming latencies in L1 after L1 from the predicted naming latencies in L1 after L2; the result was then transformed back to milliseconds. The magnitude of the L2 after-effect is represented by the greyscale. The darker it gets, the bigger the L2 after-effect. White color corresponds to the absence of the L2 after-effect.

\section{DISCUSSION}

In the analyses presented in Experiment 1, we tested the prediction of the IC-b model, according to which using L2 results in inhibiting L1, and the amount of inhibition depends on the relative language balance of an individual. We predicted that the more imbalanced a bilingual is, the more inhibition will be applied over L1 lemmas when using L2. To test this prediction, we reanalyzed the data from our past experiment that showed the L2 after-effect, 
which in the current analysis was used as an index of inhibition. The L2 after-effect was measured in a blocked picture-naming task by comparing the processing costs of production in the native language (L1) when it follows production in a second language (L2), compared to when it follows the production in L1.

As reported previously (Wodniecka et al., 2020), we observed the L2 after-effect: naming new pictures in L1 after a block of L2 naming was slower than naming in L1 after another block of naming in L1. We found that the L2 after-effect was bigger for participants with high L1 activation level (as indexed by fast baseline L1 naming latencies in the first block) and for participants with low L2 activation level (as indexed by long L2 naming latencies in the first block). This pattern of results is in line with our hypothesis which predicts that the L2 after-effect depends on the relative balance of the participants, where balance is defined as the difference between L2 and L1 activation. However, because Experiment 1 was not originally designed to test this hypothesis, we ran a replication study to further explore this novel finding while addressing the limitations of Experiment 1, which we discuss in the following paragraph.

In Experiment 1, the first stage always included two blocks of naming in the L1blocks, and after 30 minutes of other tasks, the second stage was always composed of a block of naming in L2 followed by a block of naming in L1. The fixed order in that study was intentional, 7 but it might have been confounded with other factors correlated with time such as fatigue or cumulative semantic interference (Howard, Nickels, Coltheart, \& Cole-Virtue, 2006; Runnqvist, Strijkers, Alario, \& Costa, 2012; see Wolna, Szewczyk, Casado, \&

\footnotetext{
${ }^{7}$ We used a fixed order to prevent a possible confounding influence of L2 on the L1 baseline block (L1 after L1). As it is unknown how long the effects of L2 on the subsequent L1 last, the only way to control for this possibility was to get a baseline measure (L1 after L1) before the L2 was first used in the experiment.
} 
Wodniecka, 2020, for discussion). As a consequence, the participants could have been slower to name pictures in L1 in the second testing stage (in which the L2 after-effect was measured) not because it followed a block of naming in L2 but simply because the condition in which they name in L1 after naming in L2 (L1 after L2) was always completed later in the experiment than the condition in which they name in L1 after naming in L2 (L1 after L1). In addition, the fixed order might have induced expectations towards the use of L2 in the second testing stage. In order words, the mere fact that the participants expected that at some point of the task they would need to use their L2 could have put the participants into "bilingual mode" (Grosjean, 2001) and triggered preparation for L2. This, in turn, could result in an increase of naming latencies in the L1 blocks that precede the second stage and therefore affect the baseline measurement (first block of L1 naming). Altogether, the expectations of using L2 may have modulated the measurement of language balance and perhaps also the magnitude of the L2 after-effect.

Finally, another aspect of the design of Experiment 1 that could have influenced the results is the inclusion of repeated items. Although in the presented analyses we excluded the repeated items, it is unclear how the mixing of repeated and new items may affect lexical access to the names of new pictures. Therefore, in Experiment 2, to allow purer assessment of the inhibition of the whole-language, we included only new items in the task design.

\section{EXPERIMENT 2}

Experiment 2 was specifically designed to explore how the balance between languages modulates the magnitude of the L2 after-effect. In order to overcome the limitations caused by the fixed order of sessions, in Experiment 2 we divided the testing into two sessions that were completed on separate days. To further control for possible confounds related to expectations 
regarding L2 use, the order of testing stages was counterbalanced, and we introduced a double-blinded procedure so that neither the participant nor the experimenter were aware of the appearance of L2 in the ongoing testing session (for details, see Procedure).

To fully test the hypothesis that the L1 after-effect operates on the whole-language level, each trial included a unique, never repeated item. Finally, we used a different database of pictures than in Experiment 1 in order to generalize the findings not only across participants but also across study materials.

However, running the two experimental sessions on separate days required us to control for another potential confound: day-to-day variability in the processing speed of each participant. It has been shown that participants' reaction times fluctuate from day to day (e.g., Rabbitt et al., 2001), which could confound the measurement of the L2 after-effect. First, we included the session number as a covariate in the main statistical model. Moreover, each session began with a non-linguistic choice-reaction task. This provided us with an estimate of mean reaction time in the choice-reaction task on a given day which we could subsequently include as a covariate in the main statistical model and to statistically adjust for day-to-day variability in processing speed.

\section{Participants.}

Fifty-five Polish-English bilinguals recruited via an online advertisement took part in the experiment and received monetary remuneration for their participation. Following the preregistered criteria, to qualify for the study the participants needed to have a minimum level of proficiency in L2 (English), as assessed by online tests: at least $60 \%$ on LexTALE score (Lemhöfer \& Broersma, 2012) and 18/25 points or higher in General English Test (Cambridge assessment: https://www.cambridgeenglish.org/test-your-english/general- 
english/). To ensure that the participant sample included only individuals who had high naming accuracy, we excluded 5 participants due to low naming accuracy in L1 (1 participant, less than $90 \%$ across all L1 blocks) or in L2 (4 participants, less than $60 \%$ in the L2 block). The final number of participants was established using a stopping rule (see www.osf.io/ $27 \mathrm{y} 4 \mathrm{p}$ ), according to which the precision of the L2 after-effect measurement (whether it had reached a desired standard, i.e. SE $<0.012$ ) was checked after 30 participants, and repeated after every other 5 participants. The data collection was set to stop after the stopping rule criteria were met or the data for 50 participants had been collected. The criterion for the stopping rule was based on the results of our previous experiment (Wodniecka et al., 2020) and was preregistered (see the preregistration protocol for further information: www.osf.io/ 27y4p). The final sample included 50 participants, each of which was tested in two sessions. Background information on participants' self-assessed language proficiency, learning history, and extent of language use were collected with a language background questionnaire based on the L2 Language History Questionnaire (Li et al., 2006). All participants were languageunbalanced Polish-English bilinguals whose only language acquired in early childhood was Polish (mean age of acquisition of L2 $=7.47$ years old). They started using English more intensively in secondary school at around the age of 12. Additionally, they learned another foreign language (L3; predominantly German, French or Spanish), but their L3 proficiency was low and they did not speak L3 outside the classroom context. All participants reported mostly speaking L1 during an average day and speaking L2 only occasionally. Similarly, participants considered their L1 proficiency as significantly higher than L2. To obtain an objective L2 proficiency measure, we also asked participants to fill in the Lexical Test for Advanced Learners of English, LexTALE (Lemhöfer \& Broersma, 2012). On average, 
participants obtained a score of $72 \%(\mathrm{SD}=20 \%)$, indicating that they were proficient in their L2 (for more details see Table 1).

\section{Choice Reaction Time task.}

In order to control for the baseline reaction speed in a given session, the participants performed a Choice Reaction Time task (CRT, adapted from: https:// www.cambridgecognition.com/cantab/cognitive-tests/choice-reaction-time-crt/) at the beginning of each experimental session. During each trial, an arrow appeared on the computer screen. The direction of the arrow always corresponded with its position in the screen (e.g., an arrow pointing to the right on the right side of the screen). The participants' task was to indicate the direction and position of each arrow by pressing the response buttons on the keyboard as soon as possible. The task consisted of 10 training stimuli followed by 200 target stimuli. Each trial started with a blank screen displayed for $1000 \mathrm{~ms}$, followed by $500 \mathrm{~ms}$ of a fixation point. Subsequently an arrow was presented for a maximum of $3000 \mathrm{~ms}$ for the participants to respond. The position and orientation of the arrows was randomized, with an equal number of arrows pointing to the right and to the left side. The total duration of the task was approximately 10 minutes.

\section{Picture-naming task.}

Right after the CRT task, the participants performed the picture-naming task. To control for the possible effect of the participants' expectations, a double-blind procedure was implemented at the level of experimental instructions. The experimenter explained to the participants that the task was composed of 3 experimental blocks in each session. In each block they would see various pictures in the center on the screen. Their task was to name the 
pictures aloud, and depending on the instructions displayed on the screen before each experimental block, they would have to use either Polish (L1) or English (L2). The participants were told that the language of naming was randomly assigned to each block by a computer. In such a way, the languages to be used as well as their order within the experiment were unpredictable for the participants. Additionally, the experimenter did not know which of the two sessions would be performed. We selected a total of 342 pictures from the CLT database (Haman et al., 2017), including pictures representing objects (as in Experiment 1) and pictures representing actions corresponding to verbs; pictures whose names were PolishEnglish cognates were excluded. We divided the pictures into 6 blocks of items which included 20 pictures representing actions and 37 pictures representing objects. Within each block, actions were always displayed before objects, and within each category pictures were presented randomly. We also selected 16 additional pictures to create four training blocks that were presented before the first and the second picture-naming block of each experimental session (see Figure 3). Each training block consisted of 4 pictures presented in a fixed order: 2 pictures representing actions and 2 pictures representing objects. The pictures were presented using DMDX software (Forster \& Forster, 2003) in the middle of the screen for $2000 \mathrm{~ms}$ and were preceded by a fixation cross displayed for $1000 \mathrm{~ms}$. In each session, the first and the third block required naming in L1 (Polish). The second block required naming in L1 or in L2, depending on the experimental session (see Figure 3).

\section{Data analysis}

All trials in the picture-naming task were scored for naming accuracy. Only trials with correct responses were used in the analyses. Reaction times were extracted using Chronset, which is an automated tool for detecting speech onset (Roux, Armstrong, \& Carreiras, 2017). 
The items in which naming latencies were shorter than $300 \mathrm{~ms}$ or longer than $2000 \mathrm{~ms}$ were rejected. The analysis was performed using linear mixed-effects models, as implemented in the lme4 package (version 1.1.21; Bates et al., 2015) in $\mathrm{R}$ (R Core Team, 2017), using participants and pictures as crossed random effects. For the purpose of the present experiment, we analyzed the data from blocks 2 and 3.

\section{Indices of L1 and L2 activation at Time 1}

As the indices of L1 and L2 activation at Time 1, we used the estimates of the mean latencies of naming in L1 and L2 in the second blocks of both experimental sessions. To maximize the reliability of these indices, instead of just computing the mean naming latencies for each participant, we ran two linear mixed-effects models in which the dependent variable was naming latencies in the second blocks, one model for L1 and another for L2, including bysubject and by-item random intercepts. Next, we extracted the model's per-participant estimates of naming latencies in L1 and L2 (the model's intercept, modified by the fixed effect of language and by-Participant random intercept).

\section{Index of CRT estimate}

Additionally, we calculated the estimates of response latencies in the CRT task for each session in order to control for the effect of baseline reaction speed (CRT estimate). We derived the CRT estimate separately for each session from a linear mixed model fitted for the reaction times in the CRT task with Session as a fixed factor.

\section{Main model}

In the main model, the dependent variable was the naming latencies in the third blocks of both experimental sessions (both involved naming in L1, see Figure 3). As fixed effects we included Preceding Language, Estimate of CRT, Estimate RT of L1 naming, Estimate RT of L2 naming, interaction between the Preceding Language and Estimate RT of L1 naming, and 
the interaction between the Preceding Language and Estimate RT of L2 naming. We also included a log-transformed Trial number to control for effects of familiarization, fatigue or cumulative semantic interference that could build up during the experimental session. The categorical predictor of Preceding Language was deviation coded (preceding $\mathrm{L} 1=-0.5$, preceding L2 $=0.5$ ), and the continuous predictors (Estimate of CRT, Estimate RT of L1 naming, Estimate RT of L2 naming, and Trial number) were centered prior to running the analyses. In addition, the RTs were transformed into the inverse score $(-1000 / \mathrm{RT})$ due to the right-skewed distribution of the data. We fitted the maximal model first (Barr et al., 2013), and in case of non-convergence or singularities we simplified it following the recommendations outlined in Bates and colleagues (2015). The final model included bysubject random intercept and by-item random intercept, uncorrelated by-item random slopes for Estimate RT of L1 naming, and the interaction between by-item slopes for Preceding Language and Estimate RT of L1 naming. We considered as significant any factor with absolute value of t-statistic higher than 2 .

\section{RESULTS}

Table 2 shows accuracy in all blocks of the task. Similarly to Experiment 1, participants were more accurate when naming in L1 than in L2, and the accuracy of L1 naming was the same in blocks 2 and 3, both in testing stage 1 and 2 . The analysis of naming latencies in the third blocks of both experimental sessions showed a significant effect of Preceding Language. Participants were slower to name pictures in L1 when it followed a block of naming in L2, compared to when it followed a block of naming in L1. In addition, there was a significant effect of Estimate RT of L1 naming (the faster participants named pictures in L1 in the second block, the faster they named pictures in L1 in the third blocks of 
both experimental sessions) and of the Estimate RT of L2 naming (the faster the participants named pictures in L2 in the second block, the slower they named pictures in L1 in the third blocks of both experimental sessions). Crucially, Preceding Language entered into two significant interactions: with Estimate RT of L1 naming and with Estimate RT of L2 naming (see Table 3). Both L1 and L2 baseline latencies contributed to the L2 after-effect: the faster the participants' naming in L1 (in the second block) and the slower the naming in L2 (in the second block), the larger the L2 after-effect was. In other words, the L2 after-effect was larger for participants whose naming latencies in block 2 showed the largest imbalance (i.e., they were L1 dominant; see Figure 4 for a visualization of this effect). Finally, there was an effect of Estimate of CRT, indicating that the participants that were faster in the choice-reaction task were also faster to name pictures.

\section{DISCUSSION}

Experiment 2 was designed to replicate the findings of Experiment 1 and to test if the relative language balance modulates the magnitude of the L2 after-effect. While doing so we controlled for possible confounds, namely trial-related effects like fatigue or semantic interference and the daily variability in reaction speed, which might have confounded the measurement of both the language balance index and the index of inhibition. In particular, in Experiment 2 we divided the testing stages into two experimental sessions to control for the possible effects related to the trial number (e.g., fatigue, cumulative semantic interference). We also implemented a double-blind procedure to control for participants' expectations regarding the languages to be used in the task. Additionally, in each session we included a choice reaction time task to explore and control for the role that the daily variability in the general speed of processing could have for the magnitude of the L2 after-effect. Adding these 
variables into the main model reduced the overall unexplained variance of the intercept (see Table 3), which improved the power of the statistical model.

Similarly to Experiment 1 and other previous studies (e.g.., Branzi et al., 2014), the results showed the L2 after-effect, i.e., longer naming latencies when naming in L1 followed naming in L2, compared to when naming in L1 followed naming in L1. Crucially, the magnitude of the L2 after-effect was modulated by the relative balance between L1 and L2. The results of the present experiment replicated Experiment 1: the bigger the difference between the current L2 and L1 activation levels, the higher the L2 after-effect (see Figure 4).

\section{GENERAL DISCUSSION}

In the two presented experiments, we tested an extension of the Inhibitory Control model proposed by Green (1998). More specifically, we explored the connection between the relative balance between L1 and L2 and the magnitude of L1 inhibition applied during L2 production. We tested Polish-English bilinguals with varied levels of current activation in L1 and L2. We used novel indices of an individual's magnitude of L1 inhibition and language balance. Both indices were derived from a blocked picture-naming paradigm in which participants named a set of pictures in L1 following a set of completely new pictures in either L1 or L2. To index inhibition, we used the L2 after-effect, i.e., a slow-down of L1 naming after L2 use. To index the language balance, we used a continuous measure based on baseline picture-naming latencies in L2 and in L1.

The results of both experiments provide clear evidence for the relationship between the magnitude of L1 inhibition and the relative balance between the two languages. Although most participants experienced the L2 after-effect, the effect was larger in those who were characterized by a greater imbalance between languages. These findings are in line with 
previous research that investigated differences in the magnitude of L1 inhibition in bilingual speakers but used different indices (for inhibition, the indices used in the past were derived from a language-switching paradigm; for language balance, the indices were typically based on L2 proficiency or language production accuracy, e.g., Costa \& Santesteban, 2004; Costa et al., 2006; Declerck et al., 2020 Filippi et al., 2014; Lu et al., 2019; Schweiter \& Sanderman, 2008). Besides the converging evidence supporting the main tenets of the inhibitory account, we believe that the current study provides a number of important insights into the theoretical accounts of bilingual language control as well as into the methodology used to study its underlying mechanisms.

\section{Implications for theorizing on mechanism of bilingual language control}

Initially Green proposed (1998) that whenever bilinguals want to use their second language (L2), they have to inhibit their first language in order to prevent it from interfering with L2. Building on the original IC model's assumptions, we extended the theory to propose that the amount of inhibition applied to L1 depends on the relative balance in the current activation level of the languages known by a bilingual. In the proposed IC-b model, we assume that language-unbalanced bilinguals have L2 lemmas that are less activated than L1 lemmas by default, and thus they are much more prone to interference from L1. The IC-b model further assumes that more inhibition is needed when there is a large difference between L1 and L2 baseline activation levels, namely when L1 activation level is high (i.e., L1 baseline naming latencies are relatively faster) and L2 activation level is low (i.e., L2 baseline naming latencies are relatively slower), as is in the case of unbalanced bilinguals. In contrast, when there are small differences between L1 and L2 baseline activation levels (the case of balanced bilinguals), less inhibition is needed to overcome the interference. 
The first finding of the current study is that the amount of inhibition needed to suppress L1 lemmas during L2 use is proportional to the relative balance in the activation levels of L1 and L2 lemmas in a given speaker. Because we modeled L1 and L2 activation separately, we found that they both determine the amount of inhibition applied to L1 during the use of L2, but in opposing directions. That is, the higher the activation level of L1 and the lower the activation level of L2, the bigger the L2 after-effect. The observed influence of L1 directly confirms the IC model (Green, 1998) and its claim that inhibition is reactive, i.e., the amount of inhibition applied to L1 is proportional to the baseline activation of L1. The fact that the amount of inhibition applied to L1 also depends on the activation level of L2 is, however, less obvious and extends Green's proposal to imply that the reactivity of language also depends on the activation level of the other language(s) the bilingual knows; for instance, in speakers with high baseline activation level of L2, less inhibition is applied to L1.

Interestingly, there are some reasons to think that L1 and L2 baseline activations are yoked together in the lifetime of a bilingual speaker, such as with accumulated experiences of using L2 (for example through immersion), bilinguals gradually become faster in accessing L2 at the expense of lowering the speed of accessing L1. This assumption seems in line with studies comparing bilinguals and monolinguals which show that bilinguals are overall slower to access L1 than monolinguals (for evidence from picture-naming tasks, see Gollan, Montoya, Fennema-Notestine, \& Morris, 2005; Ivanova \& Costa, 2008). Even though no longitudinal study on a bilingual population has directly shown that bilinguals gradually become faster in accessing L2 at the expense of lowering the speed of accessing L1, the results reported here allow us to speculate that as an L2 learner becomes more languagebalanced, her L2 becomes faster, and her L1 becomes proportionally slower. A comparison 
across participants with different levels of balance between L1 and L2 is visualized in Figure

5 (see also the figure in Appendix B).
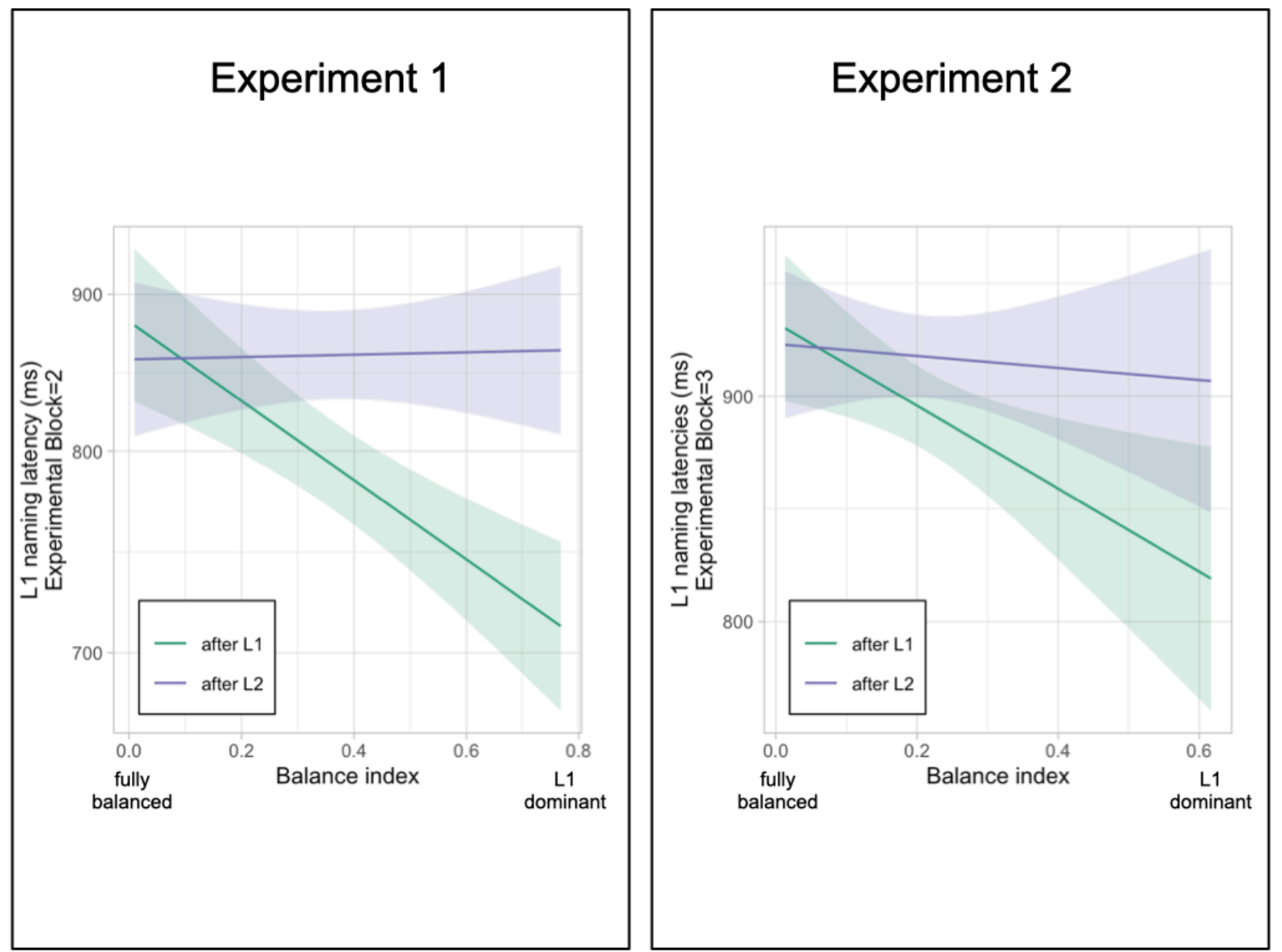

Figure 5. Predicted naming latencies in L1 after L1 and in L1 after L2 as a function of participants' language balance. The $\mathrm{x}$-axis represents the balance index, which was calculated by subtracting the predicted baseline naming latencies in L1 from the predicted baseline naming latencies in L2. The lower the value, the greater the balance between languages. The y-axis represents L1 naming latencies in the experimental blocks (Experiment 1: Block 2; Experiment 3: Block 3). Note that since language balance is defined as the difference between L1 and L2 baseline naming latencies, the same language balance can be obtained with different combinations of baseline naming latencies in L1 and L2. See Appendix B for the model's predictions for all possible combinations of L1 and L2 activation. For this graph, we selected a cross-section through these combinations such that L1 and L2 activations are in a constant trade-off (e.g. when the value of L1 baseline naming latencies decreases, the value of L2 baseline naming latencies increases by the same amount). Such a cross-section corresponds to an idealized bilingual whose baseline L2 activation increases at the expense of L1 baseline activation.

The most prominent effect visible in Figure 5 is the pattern we already discussed above: the individual language balance modulates the magnitude of the L2 after-effect (i.e., the difference between naming in L1 after L2 versus after L1). However, the figure also 
reveals a new aspect of the effect, namely that the recent use of L2 slows down L1 naming latencies to the same extent, regardless of the speaker's balance between the languages. This latter observation points to an important and curious feature of bilinguals' language system: The slow-down in L1 access observed after using L2 is likely a downstream effect of L1 inhibition that helps to achieve a level of L1 that is optimal for L2 use for a given speaker. In the case of an L1-dominant bilingual, a lot of inhibition needs to be applied to L1 in order to reach this optimal level of L1 to allow L2 access. In the case of a fully balanced bilingual, however, no L1 inhibition is needed because the baseline L1 activation is already at the optimal level to allow L2 access. In other words, fully balanced individuals appear to have the optimal level of L1 set lower, which allows them to easily access L2 at any point and prevent them from experiencing the L2 after-effect. However, on the basis of the available evidence it is impossible to tell which exact mechanism drives this reduced L1 activation. It could be a constant global L1 inhibition or simply lower default activation of L1 which allows the two languages to be kept equally accessible. In any case, the enduring down-regulation of L1 activation allows balanced bilinguals to use their two languages more efficiently on an everyday basis by adjusting their language system better to the requirements of the bilingual environment. This discovery is consistent also with the recent 'desirable difficulty' hypothesis by Kroll and colleagues (Bogulski, Bice, \& Kroll, 2019), which proposes that L2 learners need to regulate L1 and this regulation creates difficulties in L1 processing but benefits L2 learning.

The third important theoretical insight of the current study relates to the ongoing debate over the scope of language inhibition. The scope of language inhibition refers to the issue of how broadly inhibition might affect the language system, that is, whether it affects the 
language as a whole, a specific category of items, or translation equivalents. The wide scope of inhibition is usually referred to as "whole-language" (Van Assche et al., 2013) or "global" control (e.g., Abutalebi \& Green, 2016), and the narrow scope of inhibition related to translation equivalents is referred to as "item-level" (Van Assche et al., 2013) or "local" control (e.g., Abutalebi \& Green, 2016). Based on their findings, Van Assche and colleagues suggested that the ability to exert global inhibition may only develop for some types of bilinguals, whereas item-level inhibition is present in all speakers. Based on the observed pattern in a series of verbal fluency tasks, the authors claimed that whole-language inhibition was present in early Chinese-English bilinguals living in the US, but it was not present in late Dutch-English bilinguals in the Netherlands. This finding led Van Assche and colleagues to speculate that whole-language inhibition is more likely to occur when bilinguals: 1) speak structurally distinct languages, 2) acquired both languages in early childhood and in the course of their lives reversed language dominance, and 3) who live in the L2 context. However, Degani and colleagues (2020) recently tested the two scopes of inhibition (i.e., whole-language and item-level) in a group of late speakers of two similar languages (Arabic and Hebrew) and still observed whole-language inhibition in these speakers. The authors concluded that - contrary to the speculations of Van Assche and colleagues (2013) - wholelanguage inhibition is not limited to bilinguals speaking two languages as structurally distinct as Chinese and English; therefore, the language dissimilarity (as well as the early age of L2 acquisition) are unlikely to be the crucial factors that drive the engagement of whole-language inhibition. Because the Arabic-Hebrew participants were partially immersed in L2 and frequently changed languages in their daily lives, Degani and colleagues suggested that most likely the factors that engage whole-language inhibition are L2 immersion and frequent changes of languages. In contrast to this conclusion, our data indicate that whole-language 
inhibition can be systematically observed even in bilinguals whose life environment primarily involves L1. Importantly, although both our study and the one by Degani and colleagues employed a similar task to measure inhibition (i.e., picture naming), Degani and colleagues derived their measures on the basis of only error rates, whereas we used a more sensitive index based on response latencies. Moreover, unlike the investigation by Degani and colleagues, our measurement of whole-language inhibition, especially in Experiment 2, was free from additional influences of item-level inhibition as we did not repeat any items. All in all, we provide the first clear evidence that the use of a second language leads to inhibition of the native language as a whole, even in speakers who live in an L1-dominant environment ${ }^{8}$. Based on the empirical evidence discussed above, we propose that the IC-b model relates to language inhibition on the whole-language level. Whether similar effects can be observed on the item-level remains an open empirical question. However, as argued elsewhere (e.g., Van Assche et al., 2013; Wodniecka et al., 2020), probing for item-level inhibition in a picturenaming paradigm is challenging due to the processes that contaminate the measurement (e.g., picture and concept facilitation).

\section{Methodological implications for studying language inhibition in production}

We proposed and effectively used two novel indices that seem to be more ecologically valid than those used in previous research. The first index assessed a speaker's balance between the two languages known. Inspired by a solution used by Meuter and Allport (1999), we proposed that the balance can be conceptualized as the difference in the activation of L1 and L2, and it is operationalized by comparing picture-naming latencies in L1 and L2. The

\footnotetext{
8 Previous studies by Misra et al. (2012) and Guo et al. (2013), suggested that in unbalanced bilinguals living in L1 environment inhibition affects the dominant language as a whole, but the reported effects were confounded by repetition of the same pictures across experimental blocks (i.e., most likely involving item-level inhibition). Our data is free from similar impurity of the inhibition index.
} 
proposed index of balance seems to be more directly related to the levels of language activation (both default and current) than indices based on accuracy and proficiency that were used in most previous studies.

Second, we were able to demonstrate that the L2 after-effect that was derived from a blocked picture-naming paradigm can be successfully used as an index of L1 inhibition. It proved to be a robust measure which survives statistical adjustments for possible confounds in the experimental design as well as in daily variations in participants' baseline speed of processing. While controlling for these covariates is certainly important (see Experiment 2 for details), we propose that the L2 after-effect can be used as an effective index of individual strength of language inhibition in bilingual speech production. This index is sensitive enough to detect variability across speakers and to allow investigation of individual differences. Moreover, the consequences of the inhibition applied over L1 during L2 use (reflected by the L2 after-effect) are relatively long-lasting. As such, the L2 after-effect better reflects processes that take place during natural language use than the language-switching paradigm that has been used in most studies so far and which is arguably rather artificial. The L2 aftereffect is also free from some other limitations of indices derived from a language-switching paradigm, e.g. assessment of inhibition on a trial-to-trial basis (as such tapping the transient rather than long-lasting process); intense item repetition, which makes inferences about whole-language inhibition impossible; or frequent goal-switching demand, which complicates isolation of language control processes from the more domain-general demands imposed by the switching requirement.

It should be noted that the design used in the reported experiments did not allow us to investigate a possible mechanism of L2 inhibition that occurs during L1 production. Based on the IC-b model, such inhibition would indeed be expected in bilinguals with more dominant 
L2. However, here we intentionally limited our investigation to L1 inhibition to avoid contamination of the results by the previously observed effects of language testing order (for details see e.g., Guo et al., 2011; Van Assche et al., 2013). Future research should definitely explore the possibility of reverse language effects.

\section{Conclusions}

In the current paper, we explored whether individual variation in the relative balance between two languages impacts the magnitude of whole-language inhibition during language production. We proposed a causal model that explicitly outlines the assumptions of the original inhibitory account with respect to the current activation level of a bilingual's languages. In the reported experiments, we assessed the magnitude of whole-language inhibition with a novel index, namely the 'L2 after-effect', which is derived from a blocked picture-naming task. To investigate individual variation in languages' activation, we used a continuous measure of language balance that was based on naming latencies in the picturenaming task. Importantly, we kept many other potential confounding factors constant.

Our results demonstrate that individual differences in speakers' language-activation levels indeed affect the magnitude of the L2 after-effect. The more language-balanced bilinguals are, the smaller the magnitude of whole-language L1 inhibition during L2 production. In addition, we also provided some tentative evidence that language-balanced bilinguals appear to have a lower default activation level of L1 than unbalanced speakers. Future research should attempt to establish the source of this overall lower L1 activation in more balanced speakers. It is possible that the overall slower L1 performance in balanced speakers is underlined by a different mechanism than L1 inhibition applied during L2 
production. The observed dynamics in the language system may rely on multiple mechanisms of language control that are applied at different processing levels.

*Acknowledgements: The research was possible thanks to a National Science Centre grant (2015/18/E/HS6/00428) awarded to Z.W. Data collection for Experiment 1 was partially supported by National Science Centre Poland grant N106 366340 awarded to Z.W. The authors gratefully acknowledge the help of all members of our Psychology of Language and Bilingualism Laboratory LangUsta who contributed to the research project by collecting and coding the data, and Michael Timberlake with proofreading.

\section{REFERENCES}

Abutalebi, J., \& Green, D. W. (2016). Neuroimaging of language control in bilinguals: neural adaptation and reserve. Bilingualism: Language and cognition, 19(4), 689-698.

Barr, D. J., Levy, R., Scheepers, C., \& Tily, H. J. (2013). Random effects structure for confirmatory hypothesis testing: Keep it maximal. Journal of memory and language, $68(3), 255-278$.

Bates, D., Kliegl, R., Vasishth, S., \& Baayen, H. (2015). Parsimonious mixed models. arXiv preprint arXiv:1506.04967.

Bates, D., Maechler, M., Bolker, B., \& Walker, S. (2015). lme4: Linear mixed-effects models using Eigen and S4. R package version 1.1-7. 2014.

Bobb, S. C., \& Wodniecka, Z. (2013). Language switching in picture naming: What asymmetric switch costs (do not) tell us about inhibition in bilingual speech planning. Journal of Cognitive Psychology, 25(5), 568-585.

Bogulski, C. A., Bice, K., \& Kroll, J. F. (2019). Bilingualism as a desirable difficulty: Advantages in word learning depend on regulation of the dominant language. Bilingualism: Language and Cognition, 22(5), 1052-1067.

Branzi, F. M., Martin, C. D., Abutalebi, J., \& Costa, A. (2014). The after-effects of bilingual language production. Neuropsychologia, 52, 102-116.

Brysbaert, M., \& New, B. (2009). Moving beyond Kučera and Francis: A critical evaluation of current word frequency norms and the introduction of a new and 
improved word frequency measure for American English. Behavior research methods, 41(4), 977-990.

Costa, A., \& Santesteban, M. (2004). Lexical access in bilingual speech production: Evidence from language switching in highly proficient bilinguals and L2 learners. Journal of memory and Language, 50(4), 491-511.

Costa, A., Santesteban, M., \& Ivanova, I. (2006). How do highly proficient bilinguals control their lexicalization process? Inhibitory and language-specific selection mechanisms are both functional. Journal of Experimental Psychology: Learning, Memory, and Cognition, 32(5), 1057.

Declerck, M., Kleinman, D., \& Gollan, T. H. (2020). Which bilinguals reverse language dominance and why?. Cognition, 204, 104384.

Declerck, M., \& Philipp, A. M. (2015). A sentence to remember: Instructed language switching in sentence production. Cognition, 137, 166-173.

Declerck, M., \& Philipp, A. M. (2018). Is inhibition implemented during bilingual production and comprehension? n-2 language repetition costs unchained. Language, Cognition and Neuroscience, 33(5), 608-617.

Declerck, M., Thoma, A. M., Koch, I., \& Philipp, A. M. (2015). Highly proficient bilinguals implement inhibition: Evidence from n-2 language repetition costs. Journal of Experimental Psychology: Learning, Memory, and Cognition, 41(6), 1911.

Degani, T., Kreiner, H., Ataria, H., \& Khateeb, F. (2020). The impact of brief exposure to the second language on native language production: Global or item specific?. Applied Psycholinguistics, 41(1), 153-183.

Dijkstra, T. (2005). Bilingual visual word recognition and lexical access. Handbook of bilingualism: Psycholinguistic approaches, 178, 201.

Filippi, R., Karaminis, T., \& Thomas, M. S. (2014). Language switching in bilingual production: Empirical data and computational modelling. Bilingualism: Language and Cognition, 17(2), 294-315.

Forster, K. I., \& Forster, J. C. (2003). DMDX: A Windows display program with millisecond accuracy. Behavior research methods, instruments, \& computers, 35(1), 116-124.

Green, D. W. (1998). Mental control of the bilingual lexico-semantic system. Bilingualism: Language and cognition, 1(2), 67-81.

Gollan, T. H., Montoya, R. I., Fennema-Notestine, C., \& Morris, S. K. (2005). Bilingualism affects picture naming but not picture classification. Memory \& cognition, 33(7), 1220-1234.

Gollan, T. H., Schotter, E. R., Gomez, J., Murillo, M., \& Rayner, K. (2014). Multiple levels of bilingual language control: Evidence from language intrusions in reading aloud. Psychological science, 25(2), 585-595.1. 
Gollan, T. H., Weissberger, G. H., Runnqvist, E., Montoya, R. I., \& Cera, C. M. (2012). Self-ratings of spoken language dominance: A multi-lingual naming test (MINT) and preliminary norms for young and aging Spanish-English bilinguals. Bilingualism (Cambridge, England), 15(3), 594.

Grosjean, F. (2001). The bilingual's language modes. One mind, two languages: Bilingual language processing, 122 .

Guo, T., Liu, F., Chen, B., \& Li, S. (2013). Inhibition of non-target languages in multilingual word production: Evidence from Uighur-Chinese-English trilinguals. Acta psychologica, 143(3), 277-283.

Haman, E., Łuniewska, M., Hansen, P., Simonsen, H. G., Chiat, S., Bjekić, J., ... \& Gagarina, N. (2017). Noun and verb knowledge in monolingual preschool children across 17 languages: Data from cross-linguistic lexical tasks (LITMUS-CLT). Clinical linguistics \& phonetics, 31(11-12), 818-843.

Howard, D., Nickels, L., Coltheart, M., \& Cole-Virtue, J. (2006). Cumulative semantic inhibition in picture naming: Experimental and computational studies. Cognition, 100(3), 464-482.

Iniesta, A., Paolieri, D., Serrano, F., Bajo, M. (2020). Bilingual writing coactivation: lexical and sublexical processing in word dictation task. [manuscript under revision] Mind, Brain, and Behavioral Research Center, University of Granada.

Ivanova, I., \& Costa, A. (2008). Does bilingualism hamper lexical access in speech production?. Acta psychologica, 127(2), 277-288.

Kleinman, D., \& Gollan, T. H. (2018). Inhibition accumulates over time at multiple processing levels in bilingual language control. Cognition, 173, 115-132.

Kroll, J. F., Bobb, S. C., \& Wodniecka, Z. (2006). Language selectivity is the exception, not the rule: Arguments against a fixed locus of language selection in bilingual speech. Bilingualism, 9(2), 119.

Lemhöfer, K., \& Broersma, M. (2012). Introducing LexTALE: A quick and valid lexical test for advanced learners of English. Behavior research methods, 44(2), 325-343.

Li, P., Sepanski, S., \& Zhao, X. (2006). Language history questionnaire: A web-based interface for bilingual research. Behavior research methods, 38(2), 202-210.

Lu, A., Wang, L., Guo, Y., Zeng, J., Zheng, D., Wang, X., ... \& Wang, R. (2019). The Roles of Relative Linguistic Proficiency and Modality Switching in Language Switch Cost: Evidence from Chinese Visual Unimodal and Bimodal Bilinguals. Journal of psycholinguistic research, 48(1), 1-18.

Mandera, P., Keuleers, E., Wodniecka, Z., \& Brysbaert, M. (2015). Subtlex-pl: subtitlebased word frequency estimates for Polish. Behavior Research Methods, 47(2), 471-483.

Marian, V., \& Spivey, M. (2003). Bilingual and monolingual processing of competing lexical items. Applied Psycholinguistics, 24(2), 173. 
Meuter, R. F., \& Allport, A. (1999). Bilingual language switching in naming: Asymmetrical costs of language selection. Journal of memory and language, 40(1), 25-40.

Misra, M., Guo, T., Bobb, S. C., \& Kroll, J. F. (2012). When bilinguals choose a single word to speak: Electrophysiological evidence for inhibition of the native language. Journal of Memory and language, 67(1), 224-237.

Pearl, J. (2018). Comments on: The tale wagged by the DAG. International journal of epidemiology, 47(3), 1002-1004.

Philipp, A. M., Gade, M., \& Koch, I. (2007). Inhibitory processes in language switching: Evidence from switching language-defined response sets. European Journal of Cognitive Psychology, 19(3), 395-416.

Rabbitt, P., Osman, P., Moore, B., \& Stollery, B. (2001). There are stable individual differences in performance variability, both from moment to moment and from day to day. The Quarterly Journal of Experimental Psychology Section A, 54(4), 981-1003.

Roux, F., Armstrong, B. C., \& Carreiras, M. (2017). Chronset: An automated tool for detecting speech onset. Behavior research methods, 49(5), 1864-1881

Runnqvist, E., Strijkers, K., Alario, F. X., \& Costa, A. (2012). Cumulative semantic interference is blind to language: Implications for models of bilingual speech production. Journal of Memory and Language, 66(4), 850-869.

Schwieter, J. W., \& Sunderman, G. (2008). Language switching in bilingual speech production: In search of the language-specific selection mechanism. The Mental Lexicon, 3(2), 214-238.

Szekely, A., Jacobsen, T., D'Amico, S., Devescovi, A., Andonova, E., Herron, D., ... \& Federmeier, K. (2004). A new on-line resource for psycholinguistic studies. Journal of memory and language, 51(2), 247-250.

Van Assche, E., Duyck, W., \& Gollan, T. H. (2013). Whole-language and item-specific control in bilingual language production. Journal of Experimental Psychology: Learning, Memory, and Cognition, 39(6), 1781.

Wodniecka, Z., Szewczyk, J., Kałamała, P., Mandera, P., \& Durlik, J. (2020). When a second language hits a native language. What ERPs (do and do not) tell us about language retrieval difficulty in bilingual language production. Neuropsychologia, 141, 107390.

Wolna, A., Szewczyk, J., Casado, A., \& Wodniecka, Z. (2020). Now you see, now you don't. How does the experimental design impact the measurement of L2 after-effect in L1 production? [manuscript in preparation] Institute of Psychology, Jagiellonian University. 


\section{Appendix A}

Session 2. Analysis of behavioral data obtained between 4 and 9 months after the first session.

Fixed and random effects from the LME model of naming latencies in the experimental block

(Block 2)

\begin{tabular}{|l|l|l|l|l|l|}
\hline Effect & Estimate & SE & $\mathrm{t}$ & $\begin{array}{l}\text { b y }-\mathrm{I} \text { t e m } \\
\text { SD }\end{array}$ & by-Subject SD \\
\hline Intercept & -1.16011 & 0.01750 & -66.308 & 0.11429 & 0.01787 \\
\hline PrecedingLang & 0.06693 & 0.00819 & -8.172 & & 0.03938 \\
\hline Estimate.RT.L1.naming & 0.66858 & 0.06035 & 11.077 & & \\
\hline Estimate.RT.L2.naming & 0.29031 & 0.07377 & 3.935 & 0.03813 & 0.3215 \\
\hline Trial.number & 0.07384 & 0.01815 & 4.069 & & 0.05027 \\
\hline $\begin{array}{l}\text { PrecedingLang:Estimate.RT } \\
\text {.L1.naming }\end{array}$ & -0.31263 & 0.07624 & -4.101 & & - \\
\hline $\begin{array}{l}\text { PrecedingLang:Estimate.RT } \\
\text {.L2.naming }\end{array}$ & 0.36615 & 0.09242 & 3.962 & & - \\
\hline
\end{tabular}

Session 3. Analysis of behavioral data obtained between 14 and 16 months after the first session. Fixed and random effects from the LME model of naming latencies in the experimental block (Block 2)

\begin{tabular}{|l|l|l|r|l|l|}
\hline Effect & Estimate & SE & t & by-Item SD & by-Subject SD \\
\hline Intercept & -1.159510 & 0.017588 & -65.927 & 0.11377 & \\
\hline PrecedingLang & 0.067003 & 0.008729 & 7.676 & 0.02917 & 0.04077 \\
\hline Estimate.RT.L1.naming & 0.678762 & 0.060492 & 11.221 & 0.14010 & \\
\hline Estimate.RT.L2.naming & 0.306225 & 0.072693 & 4.213 & & \\
\hline Trial.number & 0.070115 & 0.018568 & 3.776 & & 0.05188 \\
\hline $\begin{array}{l}\text { PrecedingLang:Estimate.RT. } \\
\text { L1.naming }\end{array}$ & -0.307967 & 0.078174 & -3.939 & & - \\
\hline $\begin{array}{l}\text { PrecedingLang:Estimate.RT. } \\
\text { L2.naming }\end{array}$ & 0.362585 & 0.096023 & 3.776 & & - \\
\hline
\end{tabular}




\section{Appendix B}

Predicted naming latencies in L1 after L1 and L1 after L2 as a function of baseline naming speed in L1 and L2 in Experiment 1 and Experiment 2. The x-axis presents the L1 baseline naming latencies (re-transformed to milliseconds) and the y-axis the L2 baseline naming latencies (re-transformed to milliseconds). The measures were taken in Block 1 for Experiment 1, and in Block 2 for Experiment 2. The color scale represents the L1 naming latencies in the Experimental block (Experiment 1 - Block 2, Experiment 2 - Block 3). The closer to red, the longer the naming latencies. The closer to blue, the faster the naming latencies. The grey transversal lines represent language balance index (baseline naming in L2 subtracted from baseline naming in L1): the first line from the left corresponds to more L1dominant participant, and as we move to the right, the lines correspond to more balanced participants. The blue transversal line indicates the section represented in Figure 5 included in the manuscript.
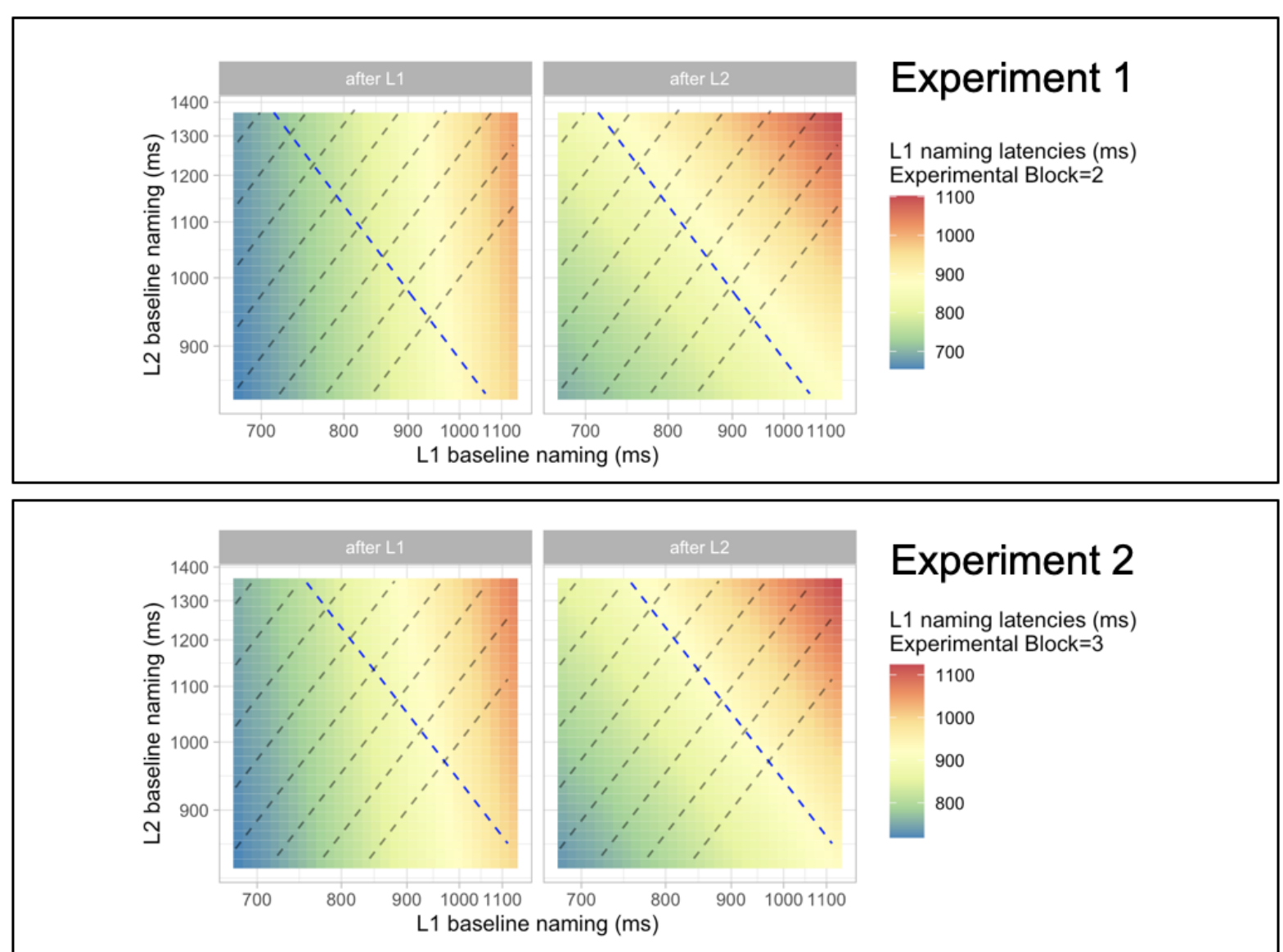